Research Article

\title{
Factors Determining Subjective Health Perception Among Middle-and Upper-Middle Aged Individuals
}

Takeshi Hatta ${ }^{1,}{ }^{*}$, Taketoshi Hatta ${ }^{2}$, Junko Hatta ${ }^{3}$, Akihiko Iwahara ${ }^{4}$, Kazumi Fujiwara ${ }^{5}$, Kimiko Kato

6 , Yukiharu Hasegawa ${ }^{1}$

1. Kansai University of Welfare Sciences, 3-11-1, Asahigaoka, Kashiwara City, Japan; E-Mails: hatta@tamateyama.ac.jp; hasegawa@tamateyama.ac.jp

2. Gifu University of Medical Sciences, Seki City, Japan; E-Mail: hatta@u-gifu-ms.ac.jp

3. Aichi Gakuin University, Nagoya City, Japan; E-Mail: hatta105@dpc.agu.ac.jp

4. Kyoto Women's University, Kyoto City, Japan; E-Mail: iwahara@kyoto-wu.ac.jp

5. Toho University, Tokyo City, Japan; E-Mail: kazumi.fujiwara@med.toho.ac.jp

6. Aichi-Syukutoku University, Nagakute City, Japan; E-Mail: kimi@asu.aasa.ac.jp

* Correspondence: Takeshi Hatta; E-Mail: hatta@tamateyama.ac.jp

Academic Editor: Calogero Caruso

Special Issue: Aging, Neuropsychology and Cognition

OBM Geriatrics

2022, volume 6, issue 1

doi:10.21926/obm.geriatr.2201190
Received: October 15, 2021

Accepted: February 17, 2022

Published: February 24, 2022

\begin{abstract}
In the present study, we identified the factors of cognitive and physical performances predicting self-rated health perception (SRH) using an SRH questionnaire in 660 (298 men and 362 women) upper-middle-aged community dwellers. In addition, neuropsychological test battery and physical ability tests were applied. A multiple regression analysis was conducted using the forced entry method; digit cancellation test (D-CAT1), logical memory test, verbal fluency test, back strength, timed-up and-go (TUG), balancing test, age, and sex were independent variables, and SRH was used as the dependent variable. The results showed that variables of D-CAT1 $(\beta=0.175, p<0.001)$, TUG $(\beta=-0.095, p<0.026)$ and body mass index (BMI; $\beta=0.095, p<0.018$ ) significantly predicted participants' SRH, whereas other variables, including sex, did not contribute significantly. D-CAT1 exerted the strongest effect on SRH.
\end{abstract}

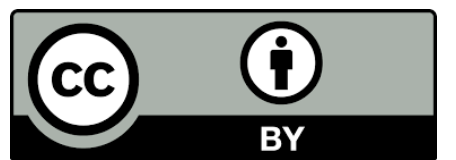

(C) 2022 by the author. This is an open access article distributed under the conditions of the Creative Commons by Attribution License, which permits unrestricted use, distribution, and reproduction in any medium or format, provided the original work is correctly cited. 
These findings indicate that upper-middle-aged individuals use their cognitive and physical performances that strongly relate to the prefrontal cortex functions to form SRH. Based on these findings, we discussed the possible effective strategies that the health-promoting staff of the local government can implement to sustain or increase the SRH of community dwellers.

\section{Keywords}

Self-rated health perception; upper-middle aged individuals; cognitive function test; physical function test; prefrontal cortex function

\section{Introduction}

Elderly individuals perform the majority of health-promoting behaviors using their subjective scale for their health, although it depends on the type of behaviors. The subjective scale is not only based on an individual's experience but also on the objective information from multiple sources of society. In addition, the elderly respect their subjective scale evaluation rather than those based on objective information, although the information is highly academic. This framework affects the health-promoting activities for the elderly recommended by the health-promoting staff. Benyamini [1] reported that self-rated health $(\mathrm{SRH})$ was strongly related to preventive health-promoting behavior. Tabernonereo et al. [2] analyzed the functions of SRH (subjective well-being and selfefficacy beliefs) in elderly patients with cardiovascular disease (CVD; mean age: 63.57 years) who answered the questionnaire assessing SRH and health-related quality of life (HRQoL). Results demonstrated that the positive evaluation of SRH was related to cardiac self-efficacy and HRQoL. These results suggested the usefulness of psychological interventions that promoted the positive affect and self-efficacy beliefs among patients with CVD. In addition, Lautenschlager et al. [3] reported that a cognitive decline in the subjective perception in old age is associated with an increased risk of future cognitive decline and dementia. Presently, no effective pharmacological treatment is available for subjective cognitive decline; the health-promoting staff of the local government needs to identify methods to enhance the subjective assessment of the elderly. Subjective health evaluation majorly depends on SRH items; for example, "how would you rate your health in general?" or "how would you rate your health compared with that of your age peers?" [4, 5]. Numerous studies have uncovered factors that contribute to the evaluation of people's health. These studies revealed that SRH sometimes predicts health outcomes more reliably than objective health-related measures [4, 6-8]. To understand the mechanisms of SRH and etiologies underlying individual differences in $\mathrm{SRH}$, several studies have been conducted using different factors such as genetic influences, developmental factors, cultural influences, evaluation methods, prediction of the onset of chronic diseases, and morbidity [1, 5, 9-13].

In the present study, we examined the cognitive and physical factors contributing to SRH of middle-aged and elderly individuals. Several previous studies have described the above-measured SRH using the Likert scale questionnaire and assessed the relationship between SRH and disease morbidity and mortality or relations between SRH and ADL (Activities of Daily Living) or IADL (Instrumental Activities of Daily Living). In addition, these studies reported that increased SRH leads to higher ADL scores [7, 9, 14]. Health psychologists use the Likert scale, i.e., a categorical scale to 
evaluate SHR $[1,5,7,9,12]$; however, the Likert scale has a shortcoming from the perspective of psychometrics. Statistics classifies variables representing data into four scale levels, namely, nominal, ordinal, interval, and ratio. The nominal scale is classified into qualitative values, whereas other quantities are classified into quantitative values. Likert scale can skew the results due to certain factors such as respondents who avoid extreme options, agree with the text presented, and exhibit themselves and their organization better. Previous studies mentioned above depend on the summation scores of nominal scales, such as ADL or IADL, and not interval or ratio scale.

We assessed the factors in cognitive and physical performances contributing to SRH using interval scale SRH measures (score: 0-100), in particular among middle-aged and elderly community residents. In case a particular cognitive factor contributes to $\mathrm{SRH}$, a special type of cognitive training must be prepared. In contrast, if a physical factor contributes to SRH, the local government's healthpromoting staff can recommend certain specially arranged physical exercises to the community dwellers. To identify the factors increasing SRH, the local government's health-promoting staff should identify specific strategies to promote health. In other words, we identified factors of cognitive or physical performance activity that contribute to the increase and maintenance of a high level of SRH, leading to corresponding special health activities. We believe that the results of the present study will contribute to reducing the financial burden on local governments.

\section{Methods}

\subsection{Participants}

The study included 680 (298 men and 362 women) community dwellers who participated in the Yakumo Study in 2012, 2013, and 2014. The Yakumo study is an ongoing cohort study that began in 1981 as a joint project between the Yakumo Town in Hokkaido and the Nagoya University Graduate School of Medicine. Research staff from several fields, such as epidemiology, internal medicine, orthopedics, urology, ophthalmology, otolaryngology, and neuropsychology, participated. The present data were selected from indices collected by the research staff of neuropsychology and orthopedics fields. The individuals participated voluntarily and could manage everyday life by themselves. Certain individuals participated annually, whereas others participated once or twice from 2012 and 2014, in which case, the first year's data were used for the analysis. One may point out that statistical processing should add the years of education of the participants as this is a desirable variable; however, when an individual over 65 years must have attended the school, the socio-economic status of the household would have determined the years of education, especially in rural areas of Japan. Therefore, we considered that educational history did not fulfill the requirements as a variable that contributed to the topics. This study was approved by the Ethics Committee of the Nagoya University Medical School for the Yakumo Study $(2011, \# 643)$ and Kansai University of Welfare Sciences (2017, \#17-13).

Participants' data were selected using the following criteria: those who answered the questionnaire without missing any items and who were examined in the neuropsychology and orthopedics sections, and their age was over 40 years. Participation in these sections was based on the free will of the participants. Participants showed no sign of any serious frailty syndrome, such as metastasized neoplastic disease, psychiatric illness, or sign of dementia as assessed by the Nagoya University Neuropsychological Assessment Battery (NU-CAB). The basic characteristics of participants are shown in Table 1. 
Table 1 Demographic data of participants.

\begin{tabular}{lll}
\hline Group & & \\
\hline Number of & Women & 362 \\
participants & Men & 298 \\
Age & Women & Mean: 62.89 (SD: 10.14) \\
& Men & Mean: 65.81 (SD: 9.51) \\
Years of & Women & Mean: 11.56 (SD: 2.42) \\
education & Men & Mean: 11.56 (SD: 2.40) \\
\hline
\end{tabular}

\subsection{Measures of SHR}

Participants completed and submitted a booklet, consisting of daily-life questions, which was distributed to their homes 3 weeks ago. An item in this questionnaire evaluated one's health status: "how would you rate your health in general on a 100-point scale." The answer to the question item was considered SRH.

\subsection{Cognitive Measures}

In the neuropsychology section, the NU-CAB was used to assess participants' cognitive abilities. The NU-CAB majorly assessed an individual's frontal cortex-related abilities, such as attention, language, memory, working memory, and executive functions [15]. The reliability and validity have been examined and reported elsewhere [16-18]. The NU-CAB consists of several test items, such as word recall test (WRT), logical memory test (LMT) (Japanese short version of Wechsler memory scale), digit cancelation test (D-CAT1 and D-CAT3), Money Road Map Test (MRMT), Stroop test, letter fluency test (LFT), and semantic fluency test (SFT). Cognitive function-related indices, D-CAT1, LMT, SFT, and LFT, were used. Using D-CAT1, we assessed the information-processing speed, focusing attention, and sustained concentration that related to the executive function of Baddeley's working memory theory. The D-CAT, developed by Hatta et al. [19], is a paper and pencil type of screening test for assessing attention and follows Sohlberg and Mateer's [20] attention model, which proposes five hierarchical classifications. Each factor of the hierarchy requires the effective functioning of the one below it. The D-CAT test sheet consisted of 12 rows of 50 digits, with each row containing five sets of numbers $0-9$, arranged randomly. In D-CAT1, participants were instructed to identify target numbers (6) and delete each one with a slash mark as fast and as accurately as possible, until the examiner sends a stop signal. The examiner stressed that all target numbers should be canceled without omission. In LMT, the examiner read twice a short story consisting of 25 segments, and each participant was asked to recall the story immediately. Each segment that was correctly recalled by the participant was assigned a score of 1 ; therefore, the total score ranged from 0 to 25 points.

Japanese SFT and LFT, standardized by Ito and Hatta [21], were administered individually. In SFT, the participants were required to produce the maximum number of nouns in $60 \mathrm{~s}$ that belonged to the assigned category's name (animal, sport, and job). In LFT, the participants were asked to generate the maximum number of noun words beginning with syllables $\mathrm{A}, \mathrm{KA}$, and $\mathrm{SHI}$, excluding proper names, numbers, and words instructed previously. After participants comprehended the instructions, the test was started. The presentation order of category names and the initial three 
letters were counterbalanced between the participants. The examiner recorded participants' aural responses.

\subsection{Physical Activity Measures}

In the orthopedics section, orthopedists conducted examinations in the presence or absence of morphological abnormalities around the knees, hips, and spine based on radiography. In addition, they measured ranges of muscle movement and bone density. Further, they measured the back strength and performed a timed up and go (TUG) test and a balance ability test using a stabilometer individually. We used the back strength measured by a back dynamometer to evaluate the muscle strength of the extensor muscle group of the trunk and the isometric strength of the hip extensor muscle group ability. The index of TUG was used to assess physical motor agility. Further, the stabilometer index and ENV AREA score were used for assessing the balancing ability.

TUG is a well-known test to measure mobility in elderly persons based on the time taken to complete a task [22, 23]. In TUG, the examiner asked the participants to perform a series of actions, such as standing up from a seated position, $3 \mathrm{~m}$ walking, turning, stopping, and sitting down. The time required to complete these tasks was measured twice by the examiner using a stopwatch. For the balancing ability, the postural tremor was measured using a stabilometer (Anima GW 5000, Anima Co., Tokyo). The manual and the standard norms were developed and normalized by Tokita [24]. Two examiners, an orthopedist, and an assistant measured the postural tremor for each participant. First, the participants stood still for $60 \mathrm{~s}$ with their eyes open, following which an eye mask was applied, and the participants were again asked to stand still for $60 \mathrm{~s}$. The participants placed their feet on the foot shapes of the device, stood upright with both arms aside, and saw a fixation point $2 \mathrm{~m}$ ahead, during which the oscillation of the center of gravity was measured. An open-eye condition ENV AREA score was used, which is the most basic parameter to assess standing body balance.

\section{$2.5 \mathrm{BMI}$}

The body mass index (BMI) was calculated using height and weight measured on the day of examination using the formula, weight/height.

\section{Statistics}

To assess whether physical and/or cognitive functions predicted $\mathrm{SRH}$, we performed a multiple regression analysis using the forced entry method, with D-CAT1, LMT, SFT, LFT, back strength, TUG, ENV AREA, age, and sex as independent variables and SRH as the dependent variable. All variables (SRH, D-CAT1, LMT, letter fluency test, semantic fluency test, back strength, TUG, ENV AREA, and $\mathrm{BMI}$ ) were normally distributed. Statistical analyses were performed using the SPSS software v.22 (IBM SPSS Statistics for Windows). We run collinearity diagnostics by inspecting the tolerance and variance inflation errors, which indicated no multicollinearity.

\section{Results}

Table 2 shows the mean performances and ranges of used measures. Table 3 shows the results of multiple regression analysis. 
The results revealed that D-CAT1, TUG, and BMI significantly predicted SRH. D-CAT1 exerted the strongest effect on SRH $(\beta=0.175, p<0.001)$, implying that the high attention-related cognitive performance related to the high SRH score. More concretely, the higher performance in the attention task was associated with a higher SRH score. In addition, TUG predicts SRH $(\beta=-0.095, p<$ 0.026), indicating faster TUG resulted in a higher SRH score. BMI was also a significant contributing factor, although the mean BMI of our sample was 23.8, and almost all participants were not obese, as in Europe and the United States. However, BMI might not be negligible for Japanese people. The factor sex failed did not significantly contribute to predicting $\mathrm{SRH}$, suggesting that the factors that create a subjective view of health are the same for both men and women, i.e., they are gender-free.

Table 2 Mean performances and ranges of used measures.

\begin{tabular}{llll}
\hline & Range & Mean & SD \\
\hline SRH & $5-100$ & 62.19 & 17.88 \\
D-CAT1 & $9-48$ & 26.58 & 6.77 \\
LMT & $0-25$ & 16.15 & 4.82 \\
Letter fluency test & $0-24$ & 8.26 & 4.33 \\
Semantic fluency test & $1-30$ & 13.47 & 5.14 \\
Back strength & $11.5-226.5$ & 76.71 & 41.26 \\
Time up and go & $2.77-35.45$ & 6.41 & 1.72 \\
ENV AREA & $0.51-13.11$ & 2.73 & 1.52 \\
BMI & $14.00-43.70$ & 23.88 & 3.47 \\
\hline
\end{tabular}

Table 3 Results of multiple regression analysis.

\begin{tabular}{|c|c|c|c|c|c|}
\hline \multirow[t]{2}{*}{ Model } & \multicolumn{2}{|c|}{ Unstandardized coefficient } & \multirow{2}{*}{$\begin{array}{l}\text { Standardized } \\
\text { coefficient } \\
\text { B }\end{array}$} & \multirow[t]{2}{*}{$t$-score } & \multirow{2}{*}{$\begin{array}{l}\text { Significant } \\
\text { probability }\end{array}$} \\
\hline & B & $\begin{array}{l}\text { Standard } \\
\text { error }\end{array}$ & & & \\
\hline Constant & 40.238 & 10.711 & & 3.757 & 0.000 \\
\hline Age & 0.075 & 0.086 & 0.042 & 0.868 & 0.386 \\
\hline D-CAT1 & 0.462 & 0.142 & 0.175 & 3.256 & 0.001 \\
\hline LMT & 0.215 & 0.161 & 0.058 & 1.340 & 0.181 \\
\hline LFT & -0.307 & 0.187 & -0.075 & -1.641 & 0.101 \\
\hline SFT & 0.063 & 0.168 & 0.018 & 0.376 & 0.707 \\
\hline TUG & -1.004 & 0.451 & -0.095 & -2.229 & 0.026 \\
\hline Back strength & -0.009 & 0.021 & -0.021 & -0.433 & 0.665 \\
\hline ENV AREA & -0.346 & 0.479 & -0.029 & -0.721 & 0.471 \\
\hline BMI & 0.487 & 0.206 & 0.095 & 2.362 & 0.018 \\
\hline Sex & -0.304 & 1.823 & -0.008 & -0.167 & 0.868 \\
\hline
\end{tabular}

\section{Discussion}

Table 3 shows that D-CAT1 and TUG were significant contributing factors to the SRH score, implying that the performances in D-CAT and TUG engaging the frontal lobe function reflected D- 
CAT1 as the most salient contributing factor to the SRH score. These findings indicate that individuals in the upper middle-age evaluated their SRH based on their subjective perceptions concerning the performances in cognitive tests, reflecting attention, information processing, and the agility of physical activities. Thus, individuals shape their SRH by interpreting cognitive and physical performances that are strongly reflected in the prefrontal cortex function.

As described earlier, D-CAT1 requires attention and rapid information processing, which is strongly reflected by the prefrontal cortex function [16, 17, 21]. In addition, it should be considered that TUG relates to the prefrontal cortex function because this task involves the planning of action sequences, sustaining physical activity plan as working memory until finally sitting down at the starting point chair. Houdeib et al. [25] examined the age-related relationship between mobility and cognitive decline using mediation analyses and reported that the cognitive factor of inhibition strongly relates to the TUG performance. This is because that TUG consists of complicated action sequences involving stopping the locomotion at a mark $10 \mathrm{~m}$ ahead, turning around, walking again, stopping locomotion, and sitting down. Because these include several inhibition elements and not a simple walking movement, executive control plays a critical role and requires the engagement of the prefrontal cortex. Compared with these two indices, the prefrontal cortex engagements in verbal fluency tasks, logical memory tasks, back strength, and ENV are relatively small or trivial.

Altogether, the present results indicated that the subjective perception of health is derived from the performances in the frontal lobe-engaged tasks. Therefore, health workers should recommend activities that reflect frontal lobe functions to increase or maintain a high SRH score.

A group of studies has examined subjective age perception (SAP) and involvement in healthpromoting activities with a concept similar to SRH $[2,3,26]$. These SAP studies used the difference between the subjective age and the calendar age as an index, ignoring the calendar age and letting the subject answer what age is now. For example, with similar purposes as those of the present study, Furuna et al. [27] examined the relationship between SAP and cognitive and physical abilities using a logistic regression analysis, where cognitive and physical abilities were independent variables, and the dependent variable was SAP. Their findings indicated that walking speed and memory test items contributed to SAP and suggested that fast walking and good memory functions help maintain a younger SAP in the elderly aged 75 years and older.

Critical differences in the present study from that of the study by Furuna et al. [27] acknowledged the contribution of memory performance (10 words delayed the recognition task), whereas the contribution of memory performance (logical memory test) in this study was negligible. In addition, they used SAP instead of SRH and employed handgrip strength, knee extension strength, standing balance, and walking speed as physical measures. However, they did not include measures that strongly related to the executive function (i.e., frontal lobe engaged functions) such as TUG, and finally, the participant's age was over 75 years. The difference in participants' age might be a contributing factor; however, it seems to be negligible. We speculate that it might be derived from the reliability of used measures. The sensitivity of the memory test due to aging was inferior to that in D-CAT [28]. In our experience, in the case of healthy subjects, the memory test did not result in a large variance as the variable, and the retest reliability were not high, although it is not the case in diagnosing patients with dementia. The reliability and sensitivity of SAP are weak, such that a certain percentage of men above 70 years old responded as 18 years old, and 50-year-old women responded as 80-year-old women [29]. Therefore, it is common in the SAP study that answers that are 2 SD or 3 SD away from the average are removed as outliers; however, this is questionable 
because all human actions should be used as data. In contrast, the SRH scale index does not produce this type of outlier; therefore, it is not necessary to artificially remove certain responses from the data analysis. In addition, the SRH index is superior to SAP. In short, present results using the interval scale SRH are more reliable than those of the study using SAP as an index.

Objective indicators have not been able to successfully encourage individuals to participate in health-promoting activities. When individuals start doubting themselves that they are obese, their subjective perception is significant rather than their evaluation of themselves as obese using an objective index, e.g., BMI. When individuals suspect themselves as subjectively obese, they start working on controlling it, such as applying dietary restrictions and attending an exercise gym. Although the objective index BMI is high, individuals do not tackle obesity as long as they subjectively judge themselves as being obese. As evident from dietary habits, an individual's subjective evaluation or perception regulates her/his lifestyle, such as brain training behavior, irrespective of the standardized cognitive test rating.

Because subjective perception strongly regulates human behavior, we evaluated the subjective health sensation on an interval scale and identified factors that contributed to SRH. We found that frontal cortex-related performances both in cognitive and physical domains regulate SRH in individuals in the upper-middle age. Next, we studied what kinds of activities should be recommended by the local government's health-promoting staff to the upper-middle-aged community dwellers. At present, tasks accompanied by not only physical movements, including agility factors but also cognitive factors with high involvement of the frontal lobe functions, are not recommended. Therefore, recommended activities should be studied separately for physical and cognitive function tasks.

An increasing number of studies have reported that physical activity is one of the strongest protective lifestyles, especially if it is maintained after middle age [30-33]. Based on the present findings, the recommended physical activity should satisfy two conditions at least; first, it should involve not only simple sequential motor actions but also agility with frequent go and not-go actions, and second, it should possess high continuity for ordinary community dwellers from the perspective of financial burden (for example, private pay training gyms or aerobic schools). Hatta et al. [30] stated that exercise habits recommended by the health-promoting staff for older individuals, such as walking and radio calisthenics, are highly unsustainable. Only $6 \%$ of the elderly have been exercising for 11 years (65-75 years). The elderly quitted from exercise habits recommended by the health-promoting staff not because their physical functions had deteriorated. The majority of the elderly people stopped exercising because these were either boring or they could not sustain the recommended exercises. Being boring is one of the common reasons observed. Therefore, it is necessary to devise a physical exercise game for the elderly that satisfies the above-mentioned two conditions.

Several previous studies have reported a crucial role in maintaining opportunities for intellectual activities for delaying the deterioration of the frontal cortex associated with aging [34]. Moreover, Hatta et al. [35] proposed that intellectual skills acquired at a later stage of human development, such as writing, and writing lyrics, among others, started to deteriorate earlier than skills acquired during childhood. Therefore, it is essential to maintain cognitive abilities acquired later in human development to delay the deterioration of the frontal lobe functions. Recent studies have reported that writing and reading are intricately related to executive functions, which are important factors of the frontal lobe functions $[36,37]$. Although the effects of sustained literacy have been proven, 
these are affected by the age-related deterioration of visual acuity. Therefore, reading habits that are less susceptible to a decrease in visual acuity and have a higher entertaining property should be considered. Examples of intellectual activities include writing short letters (although finding a pen pal and exchanging emails is fine, it is better to write it in letters); writing simple diaries; and reading newspapers, books, etc. It is possible to write by hand with large characters even if the eyesight is slightly reduced. Composing the content to be written and the act of writing letters using hand and fingers greatly engage the frontal lobe. We believe that these recommended activities require only a small financial burden. To overcome the traits of being bored of exercise and aging-related physical ability decline, it is crucial to create imaginative ideas. Several issues that could not be covered within the present study should be considered in the future. The lives of participants of this study were based on agriculture, fisheries, forestry, and working in industries and offices, and those ratios can be regarded as a typical population composition of modern Japan. However, an examination of the occupational characteristics of the target individuals and their education levels were taken into consideration, which could be a subject for future examination. In addition, comparisons with young adults and gender differences should be considered.

\section{Conclusions}

This study reported that among several cognitive and physical performance measures, D-CAT and TUG significantly predicted participants' SRH. Especially, D-CAT exerted the strongest effect on SRH. These findings indicated that individuals in the upper-middle age form their SRH based on their cognitive and physical performances that strongly relate to the functions of the prefrontal cortex. The subjective evaluation of both physical and intellectual activities leads to positive well-being. Based on these findings, the health-promoting staff of the local government should work on preparing effective strategies to sustain or increase the SRH among community dwellers.

\section{Acknowledgments}

The authors are much indebted to health promotion department of the Yakumo town for their help to conduct the study, and the first author thanks a lot to Professor Sally Carr Furnish, Manchester University, for her kind cooperation in preparing English manuscript.

\section{Author Contributions}

Both T.H. designed this study and prepared the article, and J. H., A. I., K. F., K. K., and Y. H. contributed equally to data collection and discussion.

\section{Funding}

This study was supported by grants by the JSPS KAKENHI grant number 23330219 and $17 \mathrm{H} 02647$ to the first author.

\section{Competing Interests}

The authors have declared that no competing interests exist. 


\section{References}

1. Benyamini Y. Why does self-rated health predict mortality? An update on current knowledge and a research agenda for psychologists. Psychol Health. 2011; 26: 1407-1413.

2. Tabernero $C$, Gutiérrez-Domingo $T$, Vecchione $M$, Cuadrado $E$, Castillo-Mayén R, Rubio $S$, et al. A longitudinal study on perceived health in cardiovascular patients: The role of conscientiousness, subjective wellbeing and cardiac self-efficacy. PloS ONE. 2019; 14: e0223862.

3. Lautenschlager NT, Cox KL, Ellis KA. Physical activity for cognitive health: What advice can we give to older adults with subjective cognitive decline and mild cognitive impairment? Dialogues Clin Neurosci. 2019; 21: 61-68.

4. Idler EL, Benyamini Y. Self-rated health and mortality: A review of twenty-seven community studies. J Health Soc Behav. 1997; 38: 21-37.

5. Vuorisalmi M, Lintonen T, Jylhä M. Comparative vs global self-rated health: Associations with age and functional ability. Aging Clin Exp Res. 2006; 18: 211-217.

6. Jylha M. What is self-rated health and why does it predict mortality? Towards a unified conceptual model. Soc Sci Med. 2009; 69: 307-316.

7. Latham K, Peek CW. Self-rated health and morbidity onset among late midlife US adults. J Gerontol B Psychol Sci Soc Sci. 2013; 68: 107-116.

8. McFadden E, Luben R, Bingham S, Wareham N, Kinmonth AL, Khaw KT. Does the association between self-rated health and mortality vary by social class? Soc Sci Med. 2009; 68: 275-280.

9. Christian LM, Glaser R, Porter K, Malarkey WB, Beversdorf D, Kiecolt-Glaser JK. Poorer self-rated health is associated with elevated inflammatory markers among older adults. Psychoneuroendocrinology. 2011; 36: 1495-1504.

10. Franz CE, Finkel D, Panizzon MS, Spoon K, Christensen K, Gatz M, et al. Facets of subjective health from early adulthood to old age. J Aging Health. 2017; 29: 149-171.

11. Loprinzi PD, Pazirei S, Robinson G, Dickerson B, Edwards M, Rhodes RE. Evaluation of a cognitive affective model of physical activity behavior. Health Promot Perspect. 2020; 10: 88-93.

12. Watanabe K, Kawakami N, Nishi D. Association between personal values in adolescence and mental health and well-being in adulthood: A cross-cultural study of working populations in Japan and the United States. Ann Gen Psychiatry. 2020; 19: 7.

13. Weale R. Biomarkers by gender. Arch Gerontol Geriatr. 2009; 49: 208-211.

14. Baldwin JN, McKay MJ, Hiller CE, Moloney N, Nightingale EJ, Burns J. Relationship between physical performance and self-reported function in healthy individuals across the lifespan. Musculoskelet Sci Pract. 2017; 30: 10-17.

15. Hatta T. Brain and behavior. Tokyo: Ishiyaku-syuppan; 2003.

16. Hatta T. Development of a test battery for assessment of cognitive function. J Hum Environ Stud. 2004; 2: 15-20.

17. Hatta T, Nagahara N, Iwahara A, Ito E. Three-words recall and logical memory in normal aging. J Hum Environ Stud. 2005; 3: 7-12.

18. Hatta T, Yoshizaki K, Ito Y, Mase M, Kabasawa $\mathrm{H}$. Reliability and validity of the digit cancellation test, a brief screen of attention. Psychologia. 2012; 55: 246-256.

19. Hatta T, Ito Y, Yoshizaki K. Digit Cancellation Test (D-CAT): Screening test for attention, Manual. Osaka: Union Press; 2001. 
20. Sohlberg MM, Mateer CA. Introduction to cognitive rehabilitation: Theory and practice. New York: Guilford Press; 1989.

21. Ito $E$, Hatta $T$, Ito $Y$, Kogure $T$, Watanabe H. Performance of verbal fluency tasks in Japanese healthy adults: Effect of gender, age and education on the performance. Jpn J Neuropsychol. 2004; 20: 254-263.

22. Podsiadlo D, Richardson S. The timed "Up \& Go": A test of basic functional mobility for frail elderly persons. J Am Geriatr Soc. 1991; 39: 142-148.

23. Wall JC, Bell C, Campbell S, Davis J. The Timed Get-up-and-Go test revisited: Measurement of the component tasks. J Rehabil Res Dev. 2000; 37: 109-113.

24. Tokita T. The assessment of posture tremor by stabilometer: Application and interpretation. Tokyo: Anima; 1996.

25. Langeard A, Houdeib R, Saillant K, Kaushal N, Lussier M, Bherer L. Switching ability mediates the age-related difference in timed up and go performance. J Alzheimers Dis. 2019; 71: S23-S28.

26. Stephan Y, Sutin AR, Terracciano A. "Feeling younger, walking faster": Subjective age and walking speed in older adults. Age. 2015; 37: 86.

27. Ihira H, Furuna T, Mizumoto A, Makino K, Saitoh S, Ohnishi H, et al. Subjective physical and cognitive age among community-dwelling older people aged 75 years and older: Differences with chronological age and its associated factors. Aging Ment Health. 2015; 19: 756-761.

28. Hatta $T$, Masui T, Ito $Y$, Ito E, Hasegawa Y, Matsuyama Y. Relation between the prefrontal cortex and cerebro-cerebellar functions: Evidence from the results of stabilometrical indexes. Appl Neuropsychol. 2004; 11: 153-160.

29. Hatta T, Iwahara A, Hatta T. Subjective age of Japanese middle and upper-middle healthy community dweller and their higher brain function. J Hum Environ Stud. 2009; 7: 33-42.

30. Hatta T, Kato K, Iwahara A, Hatta T, Fujiwara K, Ito E, et al. Relations between exercise habit and visual attentional ability in older adult community dwellers: Evidences from the Yakumo Study. Rehabil Process Outcome. 2018; 7: 1179572718773071.

31. Kelly ME, Loughrey D, Lawlor BA, Robertson IH, Walsh C, Brennan S. The impact of exercise on the cognitive functioning of healthy older adults: A systematic review and meta-analysis. Ageing Res Rev. 2014; 16: 12-31.

32. Loprinzi PD, Herod SM, Cardinal BJ, Noakes TD. Physical activity and the brain: A review of this dynamic, bi-directional relationship. Brain Res. 2013; 1539: 95-104.

33. Small BJ, Dixon RA, McArdle JJ, Grimm KJ. Do changes in lifestyle engagement moderate cognitive decline in normal aging? Evidence from the Victoria Longitudinal Study. Neuropsychology. 2012; 26: 144-155.

34. Cheng ST. Cognitive reserve and the prevention of dementia: The role of physical and cognitive activities. Curr Psychiatry Rep. 2016; 18: 85.

35. Hatta $T$, Ito E, Hasegawa Y. Preventing age-related cognitive decline of healthy elderly people: A preliminary report on exchanging letters method. J Hum Environ Stud. 2013; 11: 43-50.

36. Collins A, Koechlin E. Reasoning, learning, and creativity: Frontal lobe function and human decision-making. PLoS Biol. 2012; 10: e1001293.

37. Marisa F, Castro SL, Teresa L. The link between executive functions and literacy: Introduction. Read Writ. 2020; 33: 807-812. 


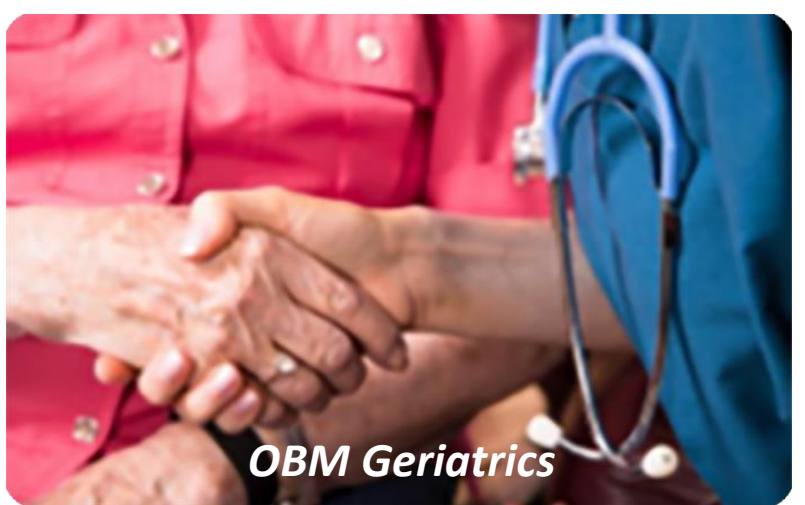

Enjoy $O B M$ Geriatrics by:

1. Submitting a manuscript

2. Joining in volunteer reviewer bank

3. Joining Editorial Board

4. Guest editing a special issue

For more details, please visit:

http://www.lidsen.com/journals/geriatrics 